\title{
Retardation in Special Relativity and the Design of a Relativistic Motor
}

\author{
A. YAhAlOM* \\ Ariel University, Kiriat Hamada POB 3, Ariel 40700, Israel
}

\begin{abstract}
The Newton third law states that any action is countered by a reaction of equal magnitude but opposite direction. The total force in a system not affected by external forces is thus zero. However, according to the principles of relativity, a signal cannot propagate at speeds exceeding the speed of light. Thus the total force cannot be null at a given time. The above conclusion leads to the possibility of a relativistic engine in particular one that is based on a permanent magnet. The analysis is based on a previous paper in which we studied the relativistic effects in a system of two current conducting loops.
\end{abstract}

DOI: 10.12693/APhysPolA.131.1285

PACS/topics: 03.30.+p, 03.50.De

\section{Introduction}

Among the major achievements of Sir Isaac Newton is the formulation of the Newton third law stating that any action is countered by a reaction of equal magnitude but opposite direction $[1,2]$. The total force in a system not affected by external forces is thus zero. This law has numerous experimental verifications and seems to be one of the cornerstones of physics. However, by the middle of the nineteenth century, Maxwell had formulated the laws of electromagnetism in his famous four partial differential equations [3-5] which were formulated in their current form by Oliver Heaviside [6]. One of the consequences of these equations is that an electromagnetic signal cannot travel at speeds exceeding that of light. This was later used by Albert Einstein [4, 5, 7] (among other things) to formulate his special theory of relativity which postulates that the speed of light is the maximal allowed velocity in nature. According to the principles of relativity, no signal (even if not electromagnetic) can propagate at superluminal velocities. Hence an action and its reaction cannot be generated at the same time because of the relativity of simultaneity. Thus the total force cannot be null at a given time. In consequence, by not holding rigorously the simultaneity of action and reaction the Newton third law cannot be held in exact form but only as an approximation. Moreover, the total force within a system that is not acted upon by an external force would not be rigorously null since the action and reactions are not able to balance each other, and the total force on a system which is not affected by an external force in not null in an exact sense.

Most locomotive systems of today are based on open systems. A rocket sheds exhaust gas to propel itself. A speeding bullet generates recoil. A car pushes the road with the same force that is used to accelerate it, the same

*corresponding author; e-mail: asya@ariel.ac.il is true regarding the interaction of a plane with air and of a ship with water. However, the above relativistic considerations suggest a new type of motor in which the open system is not composed of two material bodies but of a material body and field. Ignoring the field, a naive observer will see the material body gaining momentum created out of nothing, however, a knowledgeable observer will understand that the opposite amount of momentum is obtained by the field. Indeed, the Noether theorem dictates that any system possessing translational symmetry will conserve momentum and the total physical system containing matter and field is indeed symmetrical under translations, while every sub-system (either matter or field) is not [8]. This was already noticed by Feynman [5] (Feynman Lectures Vol. 2, 26-2 and 27-6). Feynman describes two orthogonally moving charges, apparently violating the Newton third law as the forces that the charges induce on each other do not cancel (last part of 26-2), this paradox is resolved in (27-6) in which it is shown that the momentum gained by the two charge system is balanced by the field momentum. An analogue discussion regarding retardation effects of gravitational fields was given by Carlip [9].

The purpose of this paper is to describe and analyze a relativistic engine based on a permanent magnet. Here we will use the Jefimenko equation [10] to study the force between a current loop and a permanent magnet. In this respect, the current paper differs from a previous one [11] which discussed the case of two current carrying coils, but is nevertheless based on this previous analysis. The current configuration may seem attractive for some purposes since a permanent magnet does not require a power source.

\section{Configuration}

We will assume a uniform magnetization $\boldsymbol{M}=$ $M_{z}(r, z) \boldsymbol{z}$ confined between the planes $z=-h_{1}$ and $z=-h$ such that $h<h_{1}$ and $h_{1}-h=L$. The magnet has a cylindrical shape such that the magnetization is 
confined to a radius $r \leq R_{2}$. So that

$$
M_{z}= \begin{cases}M_{0} & -h_{1} \leq z \leq-h \wedge 0 \leq r \leq R_{2}, \\ 0 & \text { otherwise. }\end{cases}
$$

In terms of the step function $u(x)$ :

$$
u(x)= \begin{cases}1 & x \geq 0 \\ 0 & x<0\end{cases}
$$

the magnetization can be written as

$$
M_{z}=M_{0}\left[u\left(z+h_{1}\right)-u(z+h)\right] u\left(R_{2}-r\right) .
$$

The magnet and a current loop above it are depicted in Fig. 1.

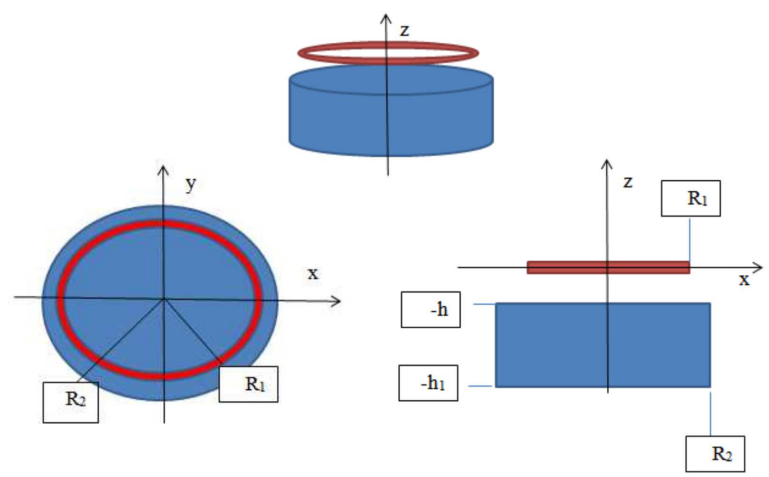

Fig. 1. A cylindrical magnet (blue) and a current loop (red) above it (three different sections).

We can now calculate the magnetization current

$$
\boldsymbol{J}_{M} \equiv \boldsymbol{\nabla} \times \boldsymbol{M} \text {. }
$$

By evaluating Eq. (4) using cylindrical coordinates

$$
\begin{gathered}
\boldsymbol{J}_{M}=\frac{1}{r}\left[\partial_{\theta}\left(M_{z}\right)-\partial_{z}\left(r M_{\theta}\right)\right] \boldsymbol{r}+\left[\partial_{z}\left(M_{r}\right)-\partial_{r}\left(M_{z}\right)\right] \boldsymbol{\theta} \\
+\frac{1}{r}\left[\partial_{r}\left(r M_{\theta}\right)-\partial_{\theta}\left(M_{r}\right)\right] \boldsymbol{z}=\frac{1}{r} \partial_{\theta}\left(M_{z}\right) \boldsymbol{r}-\partial_{r}\left(M_{z}\right) \boldsymbol{\theta} .
\end{gathered}
$$

Since $M_{z}=M_{z}(r, z)$ we obtain

$$
\boldsymbol{J}_{M}=-\partial_{r} M_{z} \boldsymbol{\theta}
$$

Moreover, since the Dirac delta function $\delta(x)$ is related to the step function by the formulae

$$
\partial_{x} u(x)=\delta(x), \quad u(x)=\int_{-\infty}^{x} \delta\left(x^{\prime}\right) \mathrm{d} x^{\prime},
$$

we obtain

$$
\begin{aligned}
& \partial_{r} u\left(R_{2}-r\right)=\frac{\partial u\left(R_{2}-r\right)}{\partial\left(R_{2}-r\right)} \frac{\partial\left(R_{2}-r\right)}{\partial r}= \\
& -\delta\left(R_{2}-r\right) .
\end{aligned}
$$

Using Eq. (8) and Eq. (3) in Eq. (6) we obtain

$$
\boldsymbol{J}_{M}=M_{0}\left[u\left(z+h_{1}\right)-u(z+h)\right] \delta\left(R_{2}-r\right) \boldsymbol{\theta} .
$$

The above can also be written in the following form:

$$
\begin{gathered}
\boldsymbol{J}_{M}=M_{0}\left[\int_{-\infty}^{z+h 1} \delta\left(z^{\prime}\right) \mathrm{d} z^{\prime}-\int_{-\infty}^{z+h} \delta\left(z^{\prime}\right) \mathrm{d} z^{\prime}\right] \delta\left(R_{2}-r\right) \boldsymbol{\theta}= \\
M_{0} \int_{z+h}^{z+h 1} \delta\left(z^{\prime}\right) \mathrm{d} z^{\prime} \delta\left(R_{2}-r\right) \boldsymbol{\theta} .
\end{gathered}
$$

$$
z^{\prime \prime}=z^{\prime}-z, \quad \mathrm{~d} z^{\prime \prime}=\mathrm{d} z^{\prime} .
$$

This will lead to

$$
\boldsymbol{J}_{M}(r, z)=M_{0} \int_{h}^{h 1} \delta\left(z^{\prime \prime}+z\right) \mathrm{d} z^{\prime \prime} \delta\left(r-R_{2}\right) \boldsymbol{\theta} .
$$

Making an additional variable change

$$
z^{\prime \prime \prime}=-z^{\prime \prime}, \quad \mathrm{d} z^{\prime \prime \prime}=-\mathrm{d} z^{\prime \prime} .
$$

results in

$$
\boldsymbol{J}_{M}(r, z)=M_{0} \int_{-h 1}^{-h} \delta\left(z-z^{\prime \prime \prime}\right) \mathrm{d} z^{\prime \prime \prime} \delta\left(r-R_{2}\right) \boldsymbol{\theta} .
$$

Hence we see that the magnetization current density is equivalent to a continuum of loop currents each with a current density

$$
\boldsymbol{J}_{M}^{(1)}\left(r, z, z^{\prime}\right) \equiv M_{0} \delta\left(z-z^{\prime}\right) \delta\left(r-R_{2}\right) \boldsymbol{\theta} .
$$

We notice that the magnetization $M_{0}$ replaces the current $I$ appearing in the analogue expression for charge current density. Hence we can write

$$
\boldsymbol{J}_{M}(r, z)=\int_{-h 1}^{-h} \boldsymbol{J}_{M}^{(1)}\left(r, z, z^{\prime}\right) \mathrm{d} z^{\prime} .
$$

\section{Force calculations}

Since in a previous paper [11] we have calculated the relativistic total force acting on a system of two coils (see equation (44) of [11]) we will take advantage of this calculation taking into account that the magnetic current density is static. For a single loop we have

$$
\boldsymbol{F}_{t}^{(1)}=\frac{\mu_{0}}{8 \pi}\left(\frac{h}{c}\right)^{2} \boldsymbol{K}_{12,2}^{(1)} I_{2} \ddot{I}_{1}=\frac{\mu_{0}}{8 \pi}\left(\frac{h}{c}\right)^{2} \boldsymbol{K}_{12,2}^{(1)} M_{0} \ddot{I}_{1},
$$

in which $\boldsymbol{K}_{(12,2)}^{(1)}$ is defined in equation (39) of [11] and we have replaced $I_{2}$ with $M_{0}, c$ is the speed of light in vacuum. The reader should notice that any current flow $I_{1}$ will result in power loss unless the current loop is superconducting. The total relativistic force is an integration over the contribution of a continuum of current loops

$$
\begin{gathered}
\boldsymbol{F}_{t}=\int_{-h 1}^{-h} \boldsymbol{F}_{t}^{(1)} \mathrm{d} z^{\prime}=\frac{\mu_{0}}{8 \pi}\left(\frac{h}{c}\right)^{2} M_{0} \ddot{I}_{1} \int_{-h 1}^{-h} \boldsymbol{K}_{12,2}^{(1)} \mathrm{d} z^{\prime} \equiv \\
\frac{\mu_{0}}{8 \pi}\left(\frac{h}{c}\right)^{2} M_{0} \ddot{I}_{1} \boldsymbol{K}_{12,2},
\end{gathered}
$$

in which $\boldsymbol{K}_{12,2}$ is an integration of the $K$ factor for all magnetization current loops. Now we wish to calculate $\boldsymbol{K}_{12,2}^{(1)}$ for this we use equation (39) of [11] which is

$$
\boldsymbol{K}_{12,2}^{(1)}\left(z^{\prime}\right)=-\frac{1}{h^{2}} \oint \oint \frac{\boldsymbol{R}}{R} \mathrm{~d} \boldsymbol{l}_{1} \cdot \mathrm{d} \boldsymbol{l}_{2} .
$$

Notice that $\boldsymbol{R}=\boldsymbol{X}_{1}-\boldsymbol{X}_{2}$ is a difference vector between the location vectors of current elements on the charge current and magnetization current loops. Hence, using suitable variables

$$
\begin{aligned}
& \boldsymbol{X}_{1}=\left(R_{1} \cos \theta_{1}, R_{1} \sin \theta_{1}, 0\right), \\
& \boldsymbol{X}_{2}=\left(R_{2} \cos \theta_{2}, R_{2} \sin \theta_{2}, z^{\prime}\right),
\end{aligned}
$$
we obtain

$$
\boldsymbol{R}=\boldsymbol{X}_{1}-\boldsymbol{X}_{2}=
$$


Thus $R$ is

$R=$

$\sqrt{\left(R_{1} \cos \theta_{1}-R_{2} \cos \theta_{2}\right)^{2}+\left(R_{1} \sin \theta_{1}-R_{2} \sin \theta_{2}\right)^{2}+z^{\prime 2}}=$

$$
\sqrt{R_{1}^{2}+R_{2}^{2}-2 R_{1} R_{2} \cos \left(\theta_{1}-\theta_{2}\right)+z^{\prime 2}} .
$$

Now we can calculate the vector line elements

$$
\begin{aligned}
& \mathrm{d} \boldsymbol{l}_{1}=R_{1} \mathrm{~d} \theta_{1} \hat{\theta_{1}}=R_{1} \mathrm{~d} \theta_{1}\left(-\sin \theta_{1}, \cos \theta_{1}, 0\right), \\
& \mathrm{d} \boldsymbol{l}_{2}=R_{2} \mathrm{~d} \theta_{2} \hat{\theta_{2}}=R_{2} \mathrm{~d} \theta_{2}\left(-\sin \theta_{2}, \cos \theta_{2}, 0\right) .
\end{aligned}
$$

The scalar product of those line elements is

$$
\mathrm{d} \boldsymbol{l}_{1} \cdot \mathrm{d} \boldsymbol{l}_{2}=R_{1} R_{2} \mathrm{~d} \theta_{1} \mathrm{~d} \theta_{2} \cos \left(\theta_{1}-\theta_{2}\right) .
$$

Combining the above results we can write the $K$ integral of Eq. (19) as

$$
\begin{aligned}
& \boldsymbol{K}_{12,2}^{(1)}\left(z^{\prime}\right)= \\
& \quad-\frac{R_{1} R_{2}}{h^{2}} \int_{0}^{2 \pi} \mathrm{d} \theta_{2} \int_{0}^{2 \pi} \mathrm{d} \theta_{1} \cos \left(\theta_{1}-\theta_{2}\right) \frac{\boldsymbol{R}\left(\theta_{1}, \theta_{2}, z^{\prime}\right)}{R\left(\theta_{1}, \theta_{2}, z^{\prime}\right)}
\end{aligned}
$$

We now make a change in variables

$$
\theta^{\prime}=\theta_{1}-\theta_{2}, \quad \mathrm{~d} \theta^{\prime}=\mathrm{d} \theta_{1} .
$$

The second equation above is correct since $\theta_{2}$ is constant for the $\theta_{1}$ integral. Hence

$$
\begin{aligned}
& \boldsymbol{K}_{12,2}^{(1)}\left(z^{\prime}\right)= \\
& \quad-\frac{R_{1} R_{2}}{h^{2}} \int_{0}^{2 \pi} \mathrm{d} \theta_{2} \int_{-\theta_{2}}^{2 \pi-\theta_{2}} \mathrm{~d} \theta^{\prime} \cos \left(\theta^{\prime}\right) \frac{\boldsymbol{R}\left(\theta^{\prime}+\theta_{2}, \theta_{2}, z^{\prime}\right)}{R\left(\theta^{\prime}+\theta_{2}, \theta_{2}, z^{\prime}\right)}
\end{aligned}
$$

In the above

$$
\frac{\boldsymbol{R}}{R}=
$$

$\frac{\left(R_{1} \cos \left(\theta^{\prime}+\theta_{2}\right)-R_{2} \cos \theta_{2}, R_{1} \sin \left(\theta^{\prime}+\theta_{2}\right)-R_{2} \sin \theta_{2},-z^{\prime}\right)}{\sqrt{R_{1}^{2}+R_{2}^{2}-2 R_{1} R_{2} \cos \theta^{\prime}+z^{\prime 2}}}$.

Now we notice that all the functions contained in the integrand of Eq. (27) are periodic in $\theta^{\prime}$ with a period of $2 \pi$ hence we can replace $\int_{-\theta_{2}}^{2 \pi-\theta_{2}} d \theta^{\prime} \rightarrow \int_{0}^{2 \pi} d \theta^{\prime}$. The following step would change the order of integrals performing the $\theta_{2}$ integral first and noticing that all the functions which are periodic in $\theta_{2}$ have a null contribution to the integral. Hence we obtain

$$
\begin{aligned}
& \boldsymbol{K}_{12,2}^{(1)}\left(z^{\prime}\right)=\frac{2 \pi R_{1} R_{2}}{h^{2}} \\
& \quad \times \int_{0}^{2 \pi} \frac{\mathrm{d} \theta^{\prime} \cos \theta^{\prime} z^{\prime}}{\sqrt{R_{1}^{2}+R_{2}^{2}-2 R_{1} R_{2} \cos \theta^{\prime}+z^{\prime 2}}} \boldsymbol{z} .
\end{aligned}
$$

Now we sum up contributions to the $K$ factor from all loops

$$
\begin{aligned}
& \boldsymbol{K}_{12,2}=\int_{-h_{1}}^{-h} \mathrm{~d} z^{\prime} \boldsymbol{K}_{12,2}^{(1)}\left(z^{\prime}\right)= \\
& \frac{2 \pi R_{1} R_{2}}{h^{2}} \int_{0}^{2 \pi} \mathrm{d} \theta^{\prime} \cos \theta^{\prime} \int_{-h_{1}}^{-h} \mathrm{~d} z^{\prime} \frac{z^{\prime}}{\sqrt{\alpha^{2}+z^{\prime 2}}} \boldsymbol{z},
\end{aligned}
$$

in which we define: $\alpha^{2} \equiv R_{1}^{2}+R_{2}^{2}-2 R_{1} R_{2} \cos \theta^{\prime}$. A simple integration leads to

$$
\boldsymbol{K}_{12,2}=\frac{2 \pi R_{1} R_{2}}{h^{2}}
$$

$$
\times \int_{0}^{2 \pi} \mathrm{d} \theta^{\prime} \cos \theta^{\prime}\left(\sqrt{\alpha^{2}+h^{2}}-\sqrt{\alpha^{2}+h_{1}^{2}}\right) z .
$$

Now we can write

$$
\begin{gathered}
\sqrt{\alpha^{2}+h^{2}}=\sqrt{h^{2}+R_{1}^{2}+R_{2}^{2}-2 R_{1} R_{2} \cos \theta^{\prime}}= \\
\sqrt{R_{1} R_{2}} \sqrt{\frac{h^{2}}{R_{1} R_{2}}+\frac{R_{1}}{R_{2}}+\frac{R_{2}}{R_{1}}-2 \cos \theta^{\prime}}
\end{gathered}
$$

and define

$$
\begin{aligned}
b & \equiv \frac{h^{2}}{R_{1} R_{2}}+\frac{R_{1}}{R_{2}}+\frac{R_{2}}{R_{1}}, \\
b_{1} & \equiv \frac{h_{1}^{2}}{R_{1} R_{2}}+\frac{R_{2}}{R_{1}}+\frac{R_{1}}{R_{2}} .
\end{aligned}
$$

Hence

$$
\begin{aligned}
& \sqrt{\alpha^{2}+h^{2}}=\sqrt{R_{1} R_{2}} \sqrt{b-2 \cos \theta^{\prime}}, \\
& \sqrt{\alpha^{2}+h_{1}^{2}}=\sqrt{R_{1} R_{2}} \sqrt{b_{1}-2 \cos \theta^{\prime}} .
\end{aligned}
$$

In terms of the above definitions

$$
\begin{aligned}
& \boldsymbol{K}_{12,2}=\frac{2 \pi\left(R_{1} R_{2}\right)^{1.5}}{h^{2}}\left[g(b)-g\left(b_{1}\right)\right] \boldsymbol{z}, \\
& g(b) \equiv \int_{0}^{2 \pi} \mathrm{d} \theta^{\prime} \cos \theta^{\prime} \sqrt{b-2 \cos \theta^{\prime}} .
\end{aligned}
$$

It is obvious that when $b \gg 2$ the respective part of the integral vanishes (the same is true for $b_{1}$ ) this is evident since the integral is performed over a constant time as cosine function of period $2 \pi$. It also clear that $b$ is a sum of a factor dependent on the magnet vertical dimensions and a factor dependent on the ratio between $R_{1}$ and $R_{2}$ which we denote $s \equiv \frac{R_{2}}{R_{1}}$. The second factor is given by

$$
f(s)=s+\frac{1}{s} \text {. }
$$

It is obvious that $f(\infty)=\infty$ and $f(0)=\infty$. Moreover, $f^{\prime}(s)=1-\frac{1}{s^{2}}$ and for $f^{\prime}(s)=0$ we obtain the minimum value $s=1$ which indicates equal radii to the magnet and the current loop. For this case $b=2+\frac{h^{2}}{R_{1}^{2}}$ and for the case that the current loop is put on the magnet $b=2$ and $g(2)=-\frac{8}{3}$. For a "thick" magnet $h_{1} \rightarrow \infty$ and thus also $b_{1} \rightarrow \infty$ so that $g\left(b_{1}\right) \rightarrow-\frac{\pi}{\sqrt{b_{1}}}$ which is unfortunately a rather slow decrease. Usually the magnet does not have to be too thick only enough to make $\left|g\left(b_{1}\right)\right|<|g(b)|$. The function $g(b)$ can be written explicitly in terms of the elliptic functions $E e(m)$ and $K e(m)$ as

$$
\begin{aligned}
& g(b)= \\
& \quad \frac{1}{3}\left\{\sqrt{b-2}\left[-b E e\left(\frac{4}{2-b}\right)+(b+2) K e\left(\frac{4}{2-b}\right)\right]\right. \\
& \left.\quad+\sqrt{b+2}\left[-b E e\left(\frac{4}{2+b}\right)+(b-2) K e\left(\frac{4}{2+b}\right)\right]\right\}, \\
& E e(m) \equiv \int_{0}^{\frac{\pi}{2}} d \theta \sqrt{1-m \sin ^{2} \theta} \\
& K e(m) \equiv \int_{0}^{\frac{\pi}{2}} \mathrm{~d} \theta \frac{1}{\sqrt{1-m \sin ^{2} \theta}} .
\end{aligned}
$$




\section{Conclusion}

We have shown in this paper that in general Newton third law is not compatible with the principles of special relativity and the total force on a system of a current loop, and a permanent magnet system is not zero. Still momentum is conserved if one takes the field momentum into account.

\section{Acknowledgments}

The author acknowledges the contribution to this work of Prof. Miron Tuval who passed away on July 28, 2016 at the age of 88 . The author would like to thank Prof. Tuval for fifteen years of collaboration and many interesting discussions of scientific and technological ideas. The author and the scientific community will always cherish his memory, may he rest in peace. The author acknowledges the generous support of Elbit systems and Mr. Ophir Levinson during the preparation of this work.

\section{References}

[1] I. Newton, Philosophice Naturalis Principia Mathematica, S. Pepys, Londini 1687.

[2] H. Goldstein, C.P. Poole Jr., J.L. Safko, Classical Mechanics, 3rd ed., Pearson, Upper Saddle River (NJ) 2001.
[3] J.C. Maxwell, A dynamical theory of the electromagnetic field, Philos. Trans. R. Soc. Lond. 155, 459 (1865).

[4] J.D. Jackson, Classical Electrodynamics, 3rd ed., Wiley, New York 1999.

[5] R.P. Feynman, R.B. Leighton, M.L. Sands, Feynman Lectures on Physics, Basic Books, revised 50th anniversary edition, 2011.

[6] O. Heaviside, On the Electromagnetic Effects due to the Motion of Electrification through a Dielectric, Philos. Mag. 27, 324 (1889).

[7] A. Einstein, Zur Elektrodynamik bewegter Körper, Ann. Phys. 322, 891 (1905).

[8] M. Tuval, A. Yahalom, Eur. Phys. J. Plus 131, 374 (2016).

[9] S. Carlip, Does Gravity Travel at the Speed of Light? (1998).

[10] O.D. Jefimenko, Electricity and Magnetism, Appleton-Century Crofts, New York 1966, 2nd ed., Electret Scientific, Star City (WV) 1989.

[11] M. Tuval, A. Yahalom, Eur. Phys. J. Plus 129, 240 (2014). 